\author{
M.I. ALIYEV ${ }^{1}$ \\ A.A. KHALILOVA ${ }^{1}$ \\ D.H. ARASLY ${ }^{1}$ \\ R.N. RAHIMOV ${ }^{1, \infty}$ \\ M. TANOGLU ${ }^{2}$ \\ L. OZYUZER ${ }^{3}$
}

\section{Strain gauges of GaSb-FeGa1.3 eutectic composites}

\author{
${ }^{1}$ Institute of Physics of Azerbaijan, National Academy of Sciences, Baku, Azerbaijan \\ ${ }^{2}$ Department of Mechanical Engineering, Izmir Institute of Technology, Gulbahce Campus, Urla, \\ Izmir 35430, Turkey \\ ${ }^{3}$ Department of Physics, Izmir Institute of Technology, Gulbahce Campus, Urla, Izmir 35430, Turkey
}

\section{Received: 5 March 2004/Accepted: 26 March 2004 Published online: 10 June 2004 • (C) Springer-Verlag 2004}

ABSTRACT A needle-shaped metallic FeGa1.3 phase oriented in a specific direction and uniformly distributed within a $\mathrm{GaSb}$ matrix was grown by a vertical Bridgman method. Strain-gauge characteristics, such as strain-sensitivity coefficient $(S)$, temperature coefficient of strain sensitivity (TCS) and temperature coefficient of resistance, of $\mathrm{GaSb}$ and $\mathrm{GaSb}-\mathrm{FeGa}_{1.3}$ eutectic alloy have been investigated in the range of 200 to $400 \mathrm{~K}$ under deformation up to strains of $1.3 \times 10^{-3}$. The value of $S$ of the GaSb-FeGa $\mathrm{F}_{1.3}$ composition is measured to be $40 \pm 5$ and its TCS is about $0.2 \% \mathrm{deg}^{-1}$ when the current is perpendicular to the needles and the needles are parallel to the plane of the gauge substrate. The strain-sensitivity characteristics are linear and hysteresis free in the investigated temperature range in the aforementioned direction. It was found that $\mathrm{GaSb}-\mathrm{FeGa} \mathrm{G}_{1.3}$ based strain gauges possess better deformation characteristics than GaSb-based gauges.

PACS 72.80.Tm; 73.40.-c; 77.65.-j

\section{$1 \quad$ Introduction}

Semiconductor resistance strain gauges, which convert a mechanical stimulus into an electrical signal, are widely used in measuring stresses and strains in many components such as structural elements, machine parts and mechanical test samples $[1,2]$. Ge-, Si- and Ge-Si-based strain gauges have been widely utilized and their properties have been investigated [3-5]. However, these semiconductor sensors exhibit a high resistivity and a high strain-sensitivity coefficient as a function of temperature. Also, there is a nonlinear behavior with strain. These factors cause a number of difficulties in modeling and designing strain-gauge-type devices. Therefore, the investigation of potential semiconductor materials to be used as mechanical sensors with thermostable parameters is essential.

$\mathrm{GaSb}$ is a candidate semiconductor for the exploration of its strain effect due to its interesting band structure $[6,7]$. Although the sensors based on GaSb show a large strainsensitivity coefficient, they exhibit a significant temperature dependence of resistivity and strain-sensitivity coefficient. Interestingly, the incorporation of $\mathrm{FeGa}_{1.3}$ metallic phase in

Fax: +994-1239-59-61, E-mail: rashad@physics.ab.az the GaSb matrix causes distinctive features of the straingauge characteristics in this material. Such heterogeneous structures possess the system of a semiconductor-metal type of GaSb-FeGa alloy. In our previous study [8], microstructure, electrical and thermal properties of $\mathrm{GaSb}-\mathrm{FeGa}_{1.3}$ eutectic composition were revealed and features of electron and phonon processes were studied. In these systems, it is considered that the electrical resistivity of the semiconductormetal boundaries is affected by the amount of the deformation on the alloy. Also, by forming eutectic alloys, the interactions between atoms are weakened and the bond energy of valance electrons that is related to the essential redistribution of charge electron densities of the nearby atoms of all components on these boundaries diminishes. This may have some considerable effect on the strain-gauge characteristics of those eutectics [9]. In such systems, a microstructure with the needle-shaped metallic FeGa phase oriented in the GaSb matrix forms. Therefore, these eutectic compositions show superior plasticity and creep behavior than those for their constituents individually due to the combination of brittle and more ductile phases.

\section{$2 \quad$ Experimental}

Semiconductor GaSb with a hole concentration of about $1.7 \times 10^{17} \mathrm{~cm}^{-3}$ was made by alloying the related components in stoichiometric quantities and refining the alloy by the method of horizontal recrystallization. Additionally, the eutectic alloys of $\mathrm{GaSb}-\mathrm{FeGa}_{1.3}$ were prepared by alloying of $\mathrm{Ga}, \mathrm{Sb}$ and $\mathrm{Fe}$ using the vertical Bridgman method as described in detail elsewhere $[8,10]$. The rate of the crystallization front was set at about $1 \mathrm{~mm} / \mathrm{min}$. By employing this technique, a structure with needle-shaped metallic $\mathrm{FeGa}_{1.3}$ phase oriented in a specific direction and uniformly distributed within the GaSb matrix was obtained. Scanning electron microscopy (SEM) was employed to characterize the microstructure of the alloys. Figure 1a and b show a SEM backscattering detector image of GaSb-FeGa $\mathrm{G}_{1.3}$ showing cross sections of the sample along (a) the longitudinal and (b) the lateral direction of the needle-shaped $\mathrm{FeGa}_{1.3}$ phase using a Philips ${ }^{\mathrm{TM}}$ FEG SEM. The backscattering imaging reveals the chemical identity of the materials and shows phases with different chemical compositions. Based on SEM examinations, the oriented needles were found to have about $1-\mu \mathrm{m}$ 


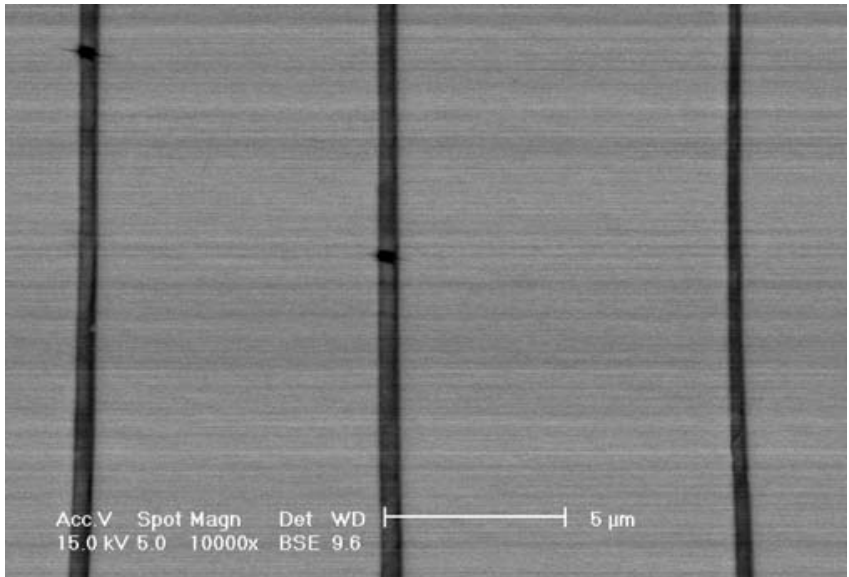

a

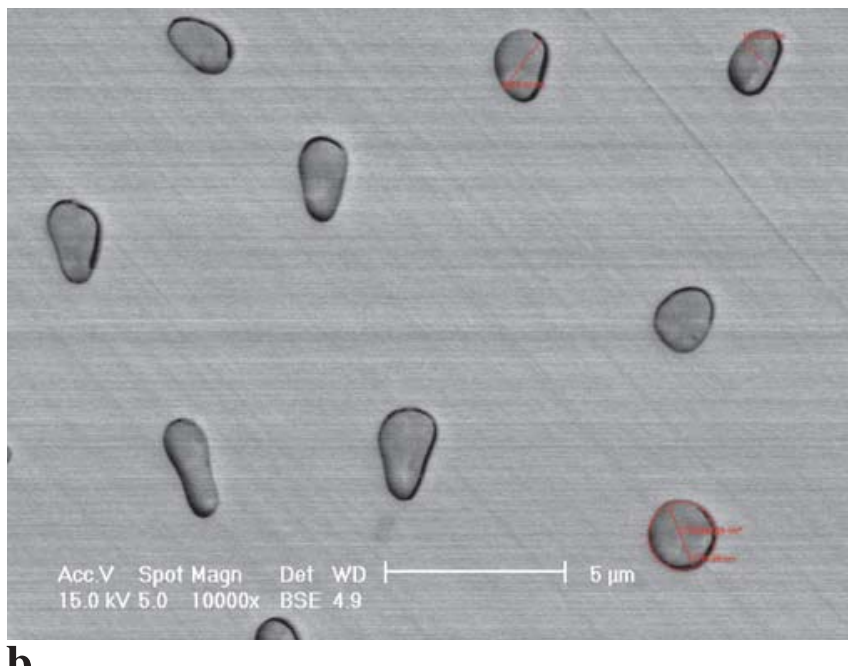

b

FIGURE 1 SEM backscattering image of $\mathrm{GaSb}-\mathrm{FeGa}_{1.3}$ showing cross section of the sample along a the longitudinal and $\mathbf{b}$ the lateral direction of the needle-shaped $\mathrm{FeGa}_{1.3}$ phase

diameter, 20-150- $\mu \mathrm{m}$ length and 4-10-microns distance from each other. The needle density $(N)$ was calculated to be about $3.3 \times 10^{4} \mathrm{~mm}^{-2}$. The obtained crystals have $p$-type conductivity with a specific resistance $\varrho=0.62 \mathrm{ohm} \mathrm{cm}$ and a hole concentration of $p \sim 10^{18} \mathrm{~cm}^{-3}$.

To determine the characteristics of the gauges, rectangular beams were sectioned from the grown crystals to obtain sensitive elements with various orientations of needles relative to the main axis of the sensor. After the mechanical and chemical treatment of the surfaces, tin contacts were applied on the gauges for electrical measurements. The contacts were ohmic, mechanically strong and reliable. It was found that Ohm's law holds true for the prepared contacts up to $30 \mathrm{~mA}$ in the range of $80 \mathrm{~K}$ to $400 \mathrm{~K}$. The produced gauges were attached on bending beams using a VL-931 glue. The thickness of the glue layer was $15 \pm 5 \mu \mathrm{m}$. The glue layer was cured at room temperature and then polymerized at elevated temperatures for $6 \mathrm{~h}$. The characterization of the strain gauges was carried out using the compensation method in the range of 200-400 K [11]. The measurements were done at three different mutual directions; $I \perp x \| P, I \perp x \perp P$ and $I\|x\| P$, where $I$ is the current, $x$ is the orientation of the metallic needles and $P$ is the bending beam that has a gauge on it.
Prior to the strain measurements the gauges were subjected to three or four heating cycles until they were stabilized. All of the studied strain gauges withstood $10^{6}$ cycles of strain at $\varepsilon=10^{-3}$ and it was observed that their strain-gauge characteristics varied with an accuracy of 5\%. The resistance and the strain-sensitivity coefficient were measured based on at least 10 samples and it was determined that the data were reproducible up to $10 \%$ deviation.

\section{3}

\section{Results and discussion}

Figure 2 shows room-temperature measurements of relative resistance versus strain characteristics at three different directions of the current, which were described above. When the direction of the current is perpendicular to the needles and the needles are parallel to the plane of the gauge substrate $(I \perp x \| P)$, the strain gauges exhibited the greatest strain-sensitivity coefficient $(S)$. It is also called the gauge factor. The value of $S$ is calculated by

$S=\frac{\Delta R / R}{\varepsilon}$.

In the $I\|x\| P$ configuration, since the needles show some continuity of the metallic phase along their longitudinal direction, the conductivity is less as compared to the other configurations. Therefore, further measurements were carried out with the $I \perp x \| P$ configuration. Figure $3 \mathrm{a}$ and $\mathrm{b}$ show measured values of the relative change in resistance $(\Delta R / R)$ for the $\mathrm{GaSb}$ matrix and $\mathrm{GaSb}-\mathrm{FeGa}_{1.3}$ composite, respectively, as a function of strain $(\varepsilon)$ for various temperatures. As is shown in the figures, there is a linear dependence of $\Delta R / R$ on both tension- and compression-type strains within the considered strain range due to the flexural bending of the substrate. The linearity does not deviate with the variation of the temperature. The dependence of $S$ on temperature for GaSb and $\mathrm{GaSb}-\mathrm{FeGa}_{1.3}$ is presented in Fig. 4 for loading under tensional and compressive loadings resulting from the bending of the substrate. The strain and temperature characteristics of the gauges do not show any hysteresis phenomenon. The

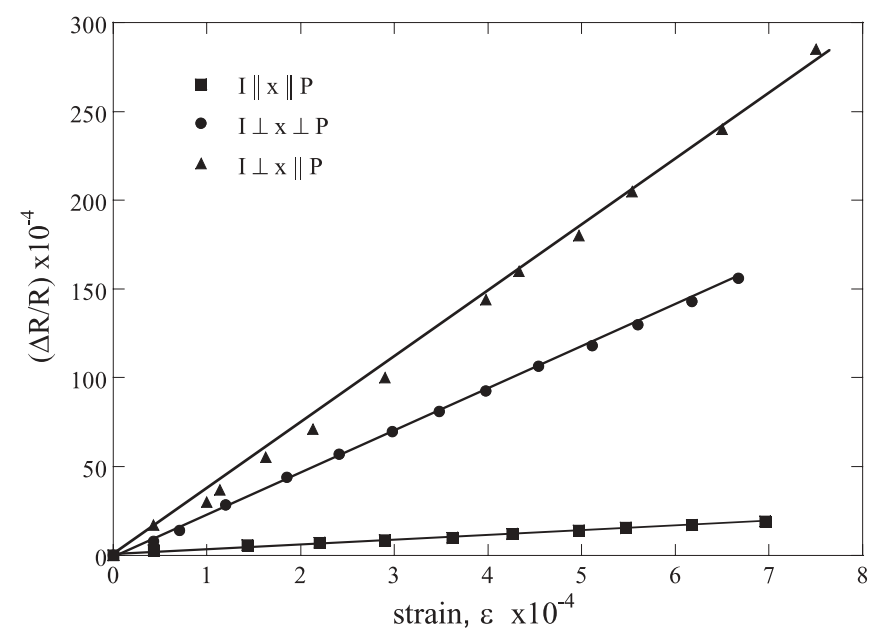

FIGURE 2 The relative change of resistance versus strain in three different directions of current $I$, needles $x$ and the plane of the sensor substrate $P$ for $\mathrm{GaSb}-\mathrm{FeGa}_{1.3}$ eutectics 

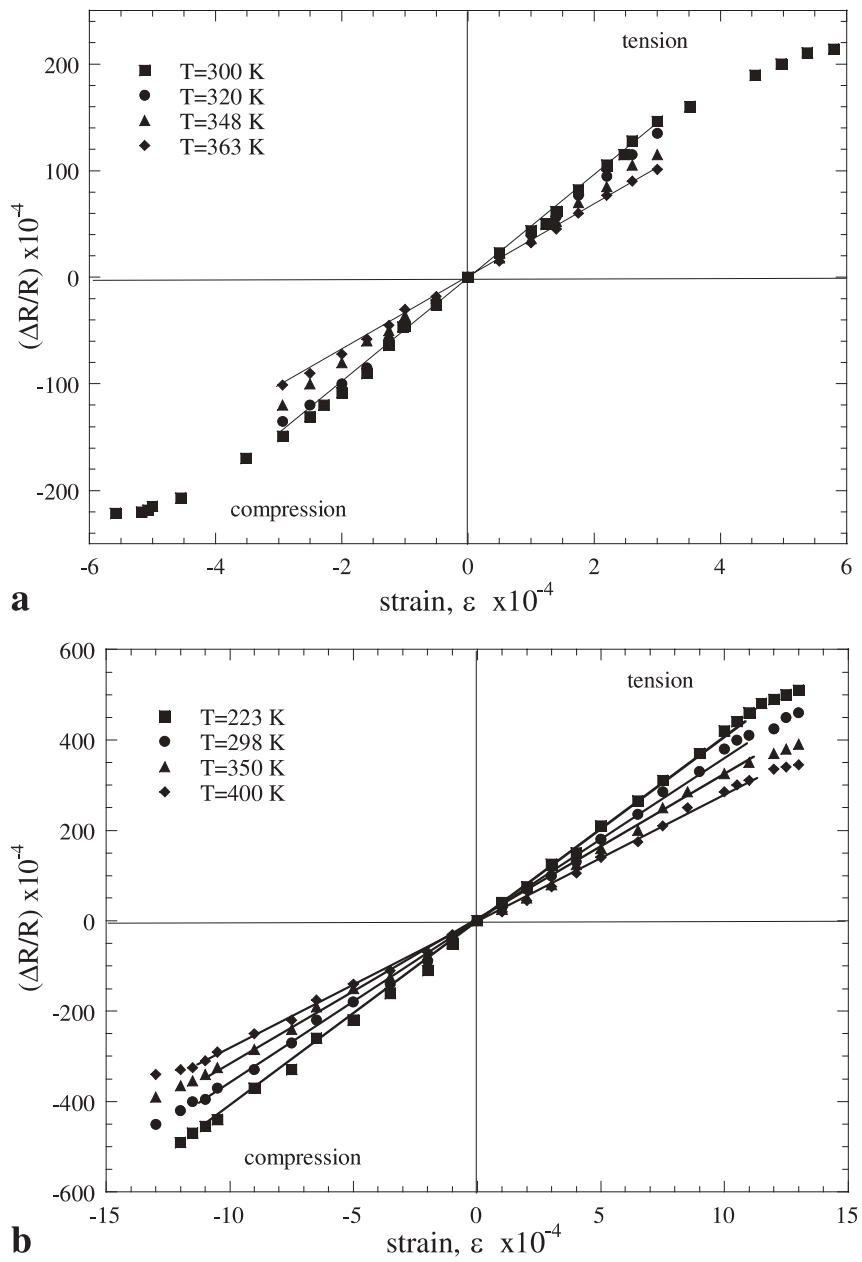

FIGURE 3 a The relative change of resistance versus strain at different temperatures for GaSb. b The relative change of resistance versus strain at different temperatures for $\mathrm{GaSb}_{-} \mathrm{FeGa}_{1.3}$ eutectics

temperature coefficients of strain sensitivity (TCS), $\alpha$, and resistance (TCR), $\beta$, were determined from the experimental data as

$\alpha=\frac{\Delta S / S_{0}}{\Delta T} \times 100\left[\% \mathrm{deg}^{-1}\right]$,

$\beta=\frac{\Delta R / R_{0}}{\Delta T} \times 100\left[\% \mathrm{deg}^{-1}\right]$,

$\Delta S=S_{T}-S_{0}, \Delta R=R_{T}-R_{0} \quad$ and $\quad \Delta T=T_{T}-T_{0}$,

where $S_{T}$ and $S_{0}\left(R_{T}\right.$ and $\left.R_{0}\right)$ are coefficients of strain sensitivity (resistance) at the fixed temperature and at room temperature, respectively. Average values of $S$ were calculated as $50 \pm 5$ and $40 \pm 5$ for $\mathrm{GaSb}$ and $\mathrm{GaSb}-\mathrm{FeGa}_{1.3}$, respectively, at room temperature. The values of $\alpha$ and $\beta$ were calculated as 0.50 and $0.30 \% \mathrm{deg}^{-1}$ for $\mathrm{GaSb}$ and 0.20 and $0.16 \% \mathrm{deg}^{-1}$ for $\mathrm{GaSb}-\mathrm{FeGa}_{1.3}$, respectively.

One of the critical parameters of the strain gauge is the limit at which the linearity of strain is conserved. This limit value was found to be about $\pm 12 \times 10^{-4}$ and $\pm 5 \times 10^{-4}$ for $\mathrm{GaSb}-\mathrm{FeGa}_{1.3}$ and $\mathrm{GaSb}$, respectively. The presented results indicate that $\mathrm{GaSb}-\mathrm{FeGa}_{1.3}$ resistance strain gauges exhibit better characteristics in comparison with $\mathrm{GaSb}$ gauges.

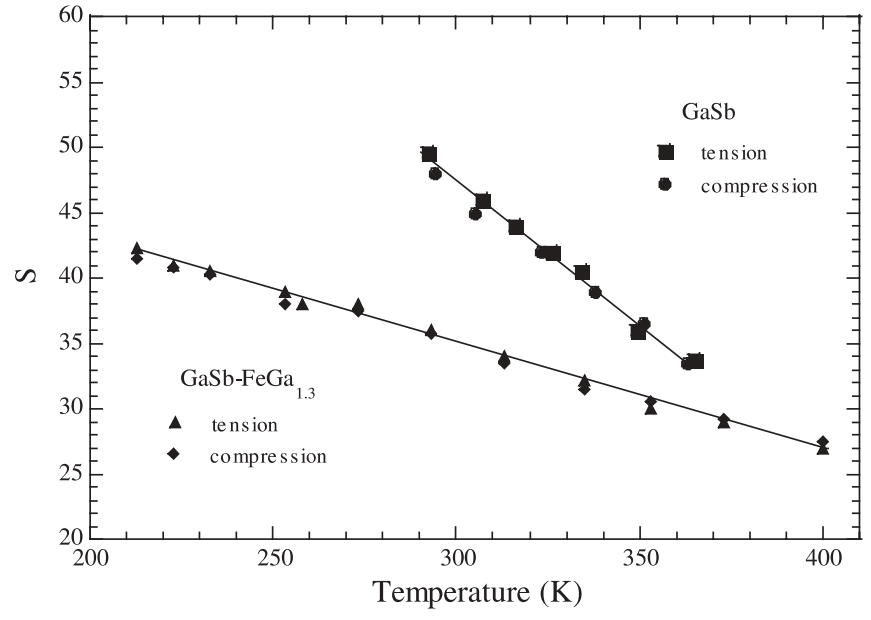

FIGURE 4 The strain-sensitivity coefficient of resistance versus temperature for $\mathrm{GaSb}$ matrix and $\mathrm{GaSb}-\mathrm{FeGa}_{1.3}$ composition

It is interesting that the $\alpha$ value of the eutectic alloy is about $60 \%$ lower than that for GaSb. It is known that in $\mathrm{GaSb}-\mathrm{FeGa}_{1.3}$ eutectics, iron atoms form deep impurity levels in the band gap of GaSb. So, the decrease of $\alpha$ values may be associated with the presence of the deep impurity levels in $\mathrm{GaSb}$.

The valence band of GaSb is degenerate in the center of the Brillouin zone $(\Gamma)$. It consists of light- and heavy-hole bands, and the third band is split due to the spin-orbit interactions [7]. The crystal symmetry under the anisotropic strain deformation is broken and it results in vanishing of degeneracy and the redistribution of holes between subbands. The valanceband edges of light and heavy holes at $\Gamma$ are displaced in opposite directions. The change of the light- and heavy-hole concentrations leads to mobility variation and gives various contributions to the conductivity. In addition, it also cause the retention of the total number of holes $(p)$, when $p=N_{\mathrm{a}}$, where $N_{\mathrm{a}}$ is the concentration of the acceptor impurity, which results in the change of the resistance. This is also true for the impurities forming slightly doped levels in the band gap when all impurity atoms are ionized at low temperatures. In addition, the small changes of impurity ionization energy under deformation (strain) do not vary the concentration of charge carriers. However, when the semiconductor material is doped by the impurity that generates the deep levels, the change of the charge-carrier concentration under deformation (strain) is significant and it cannot be neglected.

It is known that the distance between the Fermi level and deep impurity levels decreases with rise of temperature. As pointed out above, the ionization probability of deep levels increases under deformation. The value of the strain-sensitivity coefficient for each temperature is determined by the following:

$S=\frac{\mathrm{d} \varrho}{\varrho \mathrm{d} \varepsilon}=-\frac{\mathrm{d} p}{p \mathrm{~d} \varepsilon}-\frac{\mathrm{d} \mu}{\mu \mathrm{d} \varepsilon}$,

where $\varrho$ is the resistivity of the material and $p$ and $\mu$ are the concentration and the mobility of charge carriers, respectively.

Based on (1), the value of $S$ for semiconductors with deep impurity levels can be significantly below that in a material 
without the impurities. If the carrier concentration in the semiconductor changes under the deformation, the $\mathrm{d} p / p \mathrm{~d} \varepsilon$ and $\mathrm{d} \mu / \mu \mathrm{d} \varepsilon$ terms have opposite signs. However, as pointed out above, the presence of the second metallic phase generates the anisotropy in the strain-gauge characteristics. Therefore, the properties of the semiconductor matrix and the metallic inclusions must be taken into account to reveal the strain-gauge mechanisms in such heterogeneous systems. Also, the redistribution of electron charge density on the phase boundaries may be considered to have some influence on the mentioned characteristics.

\section{4}

\section{Summary}

We have shown that the incorporation of $\mathrm{FeGa}$ metallic phase in $\mathrm{GaSb}$ matrix causes distinctive features of the strain-gauge characteristics in this material. The greatest strain-sensitivity coefficient is obtained in the direction where the current is perpendicular to the needles, and the needles are parallel to the plane of the gauge substrate. The investigations of strain-sensitivity characteristics of $\mathrm{GaSb}$ matrix and $\mathrm{GaSb}-\mathrm{FeGa}_{1,3}$ composites in the range of 220 to $400 \mathrm{~K}$ have shown that their strain characteristics in this temperature range are linear and hysteresis free. The linearity limit of the strain of the $\mathrm{GaSb}-\mathrm{FeGa}_{1.3}$ eutectics is about two times larger than those in homogeneous GaSb. Also, the temperature coefficient of the strain sensitivity $(\alpha)$ and the resistance of the eutectics are, respectively, two and a half and two times less than those in homogeneous $\mathrm{GaSb}$. It is known that in $\mathrm{GaSb}-\mathrm{FeGa}_{1.3}$ eutectics, iron atoms form deep impurity levels in the band gap of GaSb. So, the decrease of $\alpha$ values may be associated with the presence of the deep impurity levels in GaSb. The presented results indicate that $\mathrm{GaSb}$ - $\mathrm{FeGa}_{1.3}$-based gauges combine in themselves the advantages of both metal and semiconductor strain gauges and exhibit better characteristics in comparison with GaSb gauges.

ACKNOWLEDGEMENTS The authors greatly acknowledge the financial support from NATO under a Collaborative Linkage Grant No. PST.CLGN 978434. L.O. acknowledges support from the Turkish Academy of Sciences, in the framework of the Young Scientist Award Program (LO/TUBA-GEBIP/2002-1-17).

\section{REFERENCES}

1 S. Middelhoek, A.A. Bellekom, U. Dauderstadt, P.J. French, S.R. in'tHout, W. Kindt, F. Riedijk, M.J. Vellekoop: Meas. Sci. Technol. 6, 1641 (1995)

2 F.Y. Obana, A.A. Carvalho, R. Gualda, J.G. da Silva: IEEE Instrumentation and Measurement Technology Conf. (Budapest, Hungary, 21-23 May 2001) 1, 429 (2001)

3 A.A. Drujinin, I.I. Maryamova, E.N. Lavitskaya, A.Q. Kutrakov, Y.M. Pankov: Datchiki Syst. 6, 2 (2001)

4 I. Maryamova, A. Druzhinin, E.N. Lavitska, I. Gortynska, Y. Yatzuk: Sens. Actuators 85, 153 (2000)

5 K. Takayama: Denshi Kagaku Electron. Sci. (Jpn.) 13, 49 (1963)

6 R.W. Keyes, M. Pollak: Phys. Rev. 118, 1001 (1960)

7 B.M. Askerov: Electron Transport Phenomena in Semiconductors (World Scientific, Singapore 1994)

8 M.I. Aliyev, A.A. Khalilova, D.H. Arasly, R.N. Rahimov, M. Tanoglu, L. Ozyuzer: J. Phys. D: Appl. Phys. 36, 2627 (2003)

9 K.Sh. Kakhramanov, V.V. Didik: Metallofizika 3, 31 (1983)

10 A. Muller, M. Wilhelm: J. Phys. Chem. Solids 26, 2029 (1965)

11 M.I. Aliyev, M. Tanoglu, A.A. Khalilova, R.N. Rahimov, D.H. Arasly: Azerb. Natl. Acad. Sci. Trans. Ser. Phys. Math. Technol. Sci. 22, 104 (2002) 